\title{
Self-reported addiction to and perceived behavioural control of waterpipe tobacco smoking and its patterns in Egypt: policy implications
}

\author{
Aya Mostafa ${ }^{1}$
}

${ }^{1}$ Department of Community, Environmental and Occupational Medicine, Faculty of Medicine, Ain Shams University, Cairo, Egypt. (Correspondence to: Aya Mostafa: aya.kamaleldin@med.asu.edu.eg).

\begin{abstract}
Background: Studies on waterpipe tobacco dependency are currently limited.

Aims: This study assessed self-reported addiction to waterpipe tobacco smoking among Egyptian waterpipe smokers and identified the associated sociodemographic factors, perceived behavioural control and patterns of waterpipe tobacco smoking.

Methods: Cross-sectional surveys were conducted on Egyptian adults in 2015 and 2017. Data on 1490 current waterpipe smokers were analysed including: sociodemographic characteristics, waterpipe tobacco smoking behaviour (age at starting, frequency, amount, company and place of smoking, and expenditure), perceived harm of waterpipe tobacco smoking, and self-reported addiction to and perceived behavioural control of waterpipe smoking (ability to quit, difficulty in quitting, quit attempts and intention to quit).

Results: A quarter (25.8\%) of the participants self-reported addiction to waterpipe tobacco smoking (males $27.1 \%$, females 11.6\%). Participants who considered themselves addicted reported less confidence in their ability to quit, fewer quit attempts, less intention to quit and less perceived harm of waterpipe smoking than those not addicted $(P<0.001)$. Variables associated with self-reported addiction were: younger age at starting waterpipe tobacco smoking (ORa $=2.2,95 \%$ CI: $1.7-$ 2.9), daily waterpipe tobacco smoking ( $\mathrm{ORa}=2.0,95 \% \mathrm{CI}$ : 1.1-3.5), smoking alone ( $\mathrm{ORa}=2.0,95 \% \mathrm{CI}: 1.4-2.8$ ), being married $(\mathrm{ORa}=1.8,95 \% \mathrm{CI}: 1.2-2.9)$, and monthly spending on waterpipe smoking of $\geq 150$ Egyptian pounds (US\$ 8.6) $(\mathrm{ORa}=4.1$, 95\% CI: 2.9-5.6).

Conclusions: Comprehensive waterpipe-specific policies are needed including education on waterpipe tobacco smoking dependency, increased taxation to decrease affordability of waterpipe tobacco and cessation programmes addressing perceived self-efficacy and addiction to waterpipe tobacco smoking.
\end{abstract}

Keywords: waterpipe tobacco smoking, behaviour, dependence, policy, Egypt

Citation: Mostafa A. Self-reported addiction to and perceived behavioural control of waterpipe tobacco smoking and its patterns in Egypt: policy implications. East Mediterr Health J. 2020;26(1):18-28. https://doi.org/10.26719/2020.26.1.18

Received: 28/02/19; accepted: 13/08/19

Copyright (C) World Health Organization (WHO) 2020. Open Access. Some rights reserved. This work is available under the CC BY-NC-SA 3.0 IGO license (https://creativecommons.org/licenses/by-nc-sa/3.o/igo).

\section{Introduction}

Waterpipe tobacco smoking is a growing global public health concern because of its associated disability, disease and compulsive use in some smokers $(1,2)$. Countries of the World Health Organization's (WHO) Eastern Mediterranean Region are at the centre of this epidemic (3). Member States, including Egypt, have agreed on a global target to achieve a $30 \%$ relative reduction in tobacco use by 2025 (4); however, the overall tobacco smoking rates in Egypt are projected to increase by at least $20 \%$ among males by this time (5). In Egypt, recent national estimates of the prevalence of current waterpipe tobacco smoking were $8.7 \%$ and $0.1 \%$ in males and females aged $15-69$ years old, respectively (6). Trends suggest this gender gap is closing. Young females are increasingly using non-cigarette tobacco products, including waterpipe tobacco, more than young males and older females $(7,8)$.

Despite this growing prevalence and documented harm, waterpipe tobacco smoking has been inadequately addressed by national tobacco control policies. The behaviour of waterpipe tobacco smokers has not been fully characterized and waterpipe tobacco is often mislabelled as an occasional method of tobacco use (compared with cigarette smoking). However, in recent population-based surveys, 50-80\% of Egyptian waterpipe smokers reported daily use of waterpipe tobacco $(6,9)$. In addition, doubts have been raised about whether waterpipe tobacco smoking leads to dependence (10). The limited amount of relevant research may have contributed to this uncertainty (1).

Research investigating factors associated with waterpipe tobacco dependence are limited. In particular, little is known about whether waterpipe smokers consider themselves addicted to waterpipe tobacco smoking. This self-identification is crucial in order to progress along the stages of behavioural change from precontemplation, 
where the smoker is in denial of their addictive smoking behaviour, towards contemplation, where the smoker starts considering smoking cessation (11). It is at this later stage that the smoker can get most benefit from cessation interventions.

It is important to characterize the context in which waterpipe smokers consider quitting because dependence is a multidimensional matter. It would help us understand this context if information were available on frequency and intensity (amount of waterpipe tobacco smoked) of waterpipe tobacco smoking (as proxy measures of dependence); price of and expenditure on waterpipe tobacco (as measures of affordability); place where waterpipe tobacco smoking takes place and is obtained (as measures for accessibility); whether waterpipe tobacco smoking is done in company or alone and type of tobacco smoked (as measures of social desirability); and perceived harm and behavioural control of waterpipe smoking (as measures of self-efficacy) (1). Few data are available on how the sociodemographic characteristics of waterpipe smokers and the patterns of waterpipe tobacco use could influence smokers' perceived behavioural control, and hence their self-identification as being dependent on waterpipe tobacco.

Examining these variables is important in order to develop and tailor evidence-based interventions to reduce waterpipe tobacco smoking. Relevant evidence may better inform comprehensive policy interventions that tackle both supply and demand measures for effective tobacco control, as recommended by the WHO Framework Convention on Tobacco Control, such as cessation, taxation and education interventions (12). The aim of this study therefore was to assess self-reported addiction to waterpipe tobacco smoking in adult Egyptian waterpipe smokers and identify the associated sociodemographic factors, waterpipe tobacco smoking behaviour, perception of harm and behavioural control of waterpipe tobacco smoking.

\section{Methods}

Specifics of the study design, sample, survey tool and procedures have been detailed previously $(8,9)$. Briefly, data included in this study are part of a study that consisted of two identical cross-sectional surveys done in July to November 2015 and September 2016 to January 2017. Participants were recruited from a purposive quota sample of 2014 waterpipe smokers and non-smokers living in Cairo and a village in the Nile Delta. Male and female participants were invited to take part in a face-to-face interview survey if they were 18 years or older. For this study, data were analysed on current waterpipe smokers only ( $n=1490,74.0 \%$ of the total sample) including participants' sociodemographic characteristics, tobacco use, exposure to household second-hand smoke, waterpipe tobacco smoking behaviour, perceived harm of waterpipe tobacco smoking, self-reported addiction to waterpipe tobacco smoking and perceived behavioural control of waterpipe tobacco smoking .

\section{Participant characteristics}

Participants' sociodemographic characteristics included: age, gender, residence, educational level, occupation, marital status and crowding index. The study also assessed whether waterpipe smokers had smoked cigarettes in the 30 days before the survey and their household exposure to second-hand smoke from cigarette smoking or waterpipe tobacco smoking.

\section{Waterpipe tobacco smoking behaviour}

Age at starting to smoke waterpipe tobacco was recorded. Current use was defined as any waterpipe tobacco smoking in the 30 days before the survey (13). Current waterpipe smokers described their usual frequency of waterpipe tobacco smoking as: monthly (at least once a month, but not weekly), weekly (at least once a week, but not daily) or daily (at least once a day or on most days of the month). The time of the last waterpipe session was also assessed (today, a couple of days ago, last week, last month). Participants reported the intensity of waterpipe tobacco smoking as number of waterpipe tobacco portions (hagar) smoked a day and in the past 30 days. The usual place where participants smoke the waterpipe (café/restaurant, at home, workplace, at a friend's place) was assessed as were their average frequency of waterpipe tobacco smoking at cafés in the past 30 days, whether they usually smoked the waterpipe alone (always, most of the time, sometimes, never) or in the company of others, and the waterpipe tobacco type they usually smoked (flavoured or unflavoured). Participants were asked about where they usually purchased waterpipe tobacco (market, street vendor, smoke shop, café or restaurant, friend or relative, Internet, other). Participants were also asked about their average daily spending on waterpipe tobacco smoking - prices are reported in Egyptian pounds and converted to American dollars (US \$) (14) - and the percentage of their monthly income they spent on waterpipe tobacco smoking ( $\leq 1 \%, 2-10 \%, 11-50 \%,>50 \%)$.

\section{Perceived harm of waterpipe tobacco smoking}

Participants were asked how often they thought about the cost of waterpipe tobacco smoking (never, sometimes, often); what effect they thought waterpipe tobacco smoking had on health in general (good, bad, neither good nor bad, don't know); how often they worried about the health hazards of waterpipe tobacco smoking (never, sometimes, often); how harmful they thought waterpipe tobacco smoking was compared with cigarettes (less harmful, about the same, more harmful, don't know); and how much nicotine was in waterpipe tobacco smoking compared with cigarettes (less nicotine, about the same, more nicotine, don't know).

\section{Self-reported addiction to and perceived behavioural control of waterpipe tobacco smoking}

To assess how current waterpipe smokers perceived their dependence on waterpipe tobacco smoking, participants were asked if they considered themselves addicted to 
waterpipe tobacco smoking (yes, no, don't know); if they were confident in their ability to quit waterpipe tobacco smoking (perceived self-efficacy) any time they wanted (yes, no); how easy they thought it would be to permanently quit waterpipe tobacco smoking (easy, difficult, don't know); whether they had any intention to quit waterpipe tobacco smoking (not at all, in the next month, in the next 6 months, in the future) and whether they had ever attempted to quit waterpipe tobacco smoking (yes, no).

\section{Statistical analysis}

For the descriptive analysis, means, standard deviations $(\mathrm{SD})$, medians and interquartile ranges (IQR) were calculated for continuous variables and proportions were calculated for categorical variables. No statistically significant differences were found in the background characteristics of current waterpipe smokers in the two surveys. Therefore, data from both rounds of the survey were combined for all current waterpipe smokers $(n=1490)$. Univariate analysis was done using the chi-squared test for categorical variables and the independent samples t-test for continuous variables to identify statistically significant associations between participants' self-reported addiction (dependent variable) and their characteristics (sociodemographic, exposure to household second-hand smoke, use of cigarettes in the past 30 days), waterpipe tobacco smoking behaviour, perceived harm of waterpipe tobacco smoking and perceived behavioural control of waterpipe tobacco smoking.

For self-reported addiction to waterpipe tobacco smoking, "don't know" answers" were considered as "no". Multivariable logistic regression analyses were done to identify independent factors associated with self-reported addiction to waterpipe tobacco smoking. The following variables were tested as independent determinants of self-reported addiction to waterpipe tobacco smoking: male gender, older age, higher educational level, employed, married, no history of cigarette smoking in the past 30 days, daily waterpipe tobacco smoking, younger age at starting waterpipe tobacco smoking, usually smoking the waterpipe at home, smoking the waterpipe alone, using unflavoured waterpipe tobacco, self-purchase of waterpipe tobacco (from a market/street vendor/smoke shop/internet) and spending 150 Egyptian pounds (US\$ 8.6) or more a month on waterpipe tobacco smoking.

All variables that were statistically significant in the univariate analysis at $P \leq 0.05$ were entered into the multivariable regression model. Adjusted odds ratios (ORa) and $95 \%$ confidence intervals (CI) are reported. The significance level was set as 0.05 . SPSS, version 25 was used for all analyses.

\section{Ethical considerations}

The study was approved by the Ethical Review Board of the Faculty of Medicine, Ain Shams University, Cairo, Egypt. Verbal informed consent to take part in the surveys was obtained from all the participants.

\section{Results}

\section{Participant characteristics and self-reported addiction to waterpipe tobacco smoking}

A quarter $(384,25.8 \%)$ of current waterpipe smokers self-reported addiction to waterpipe tobacco smoking (Table 1). Participants' sociodemographic characteristics are shown in Table 1. More than two thirds (1009, 67.7\%) of the participants were exposed to second-hand smoke at home, and almost half of the participants (663, 44.5\%) reported smoking cigarettes in the 30 days before the survey. The crowding index was average (2 to 3 ) (Table 1). Significantly more of the participants who self-reported addiction to waterpipe tobacco smoking than those who did not were male, older, had a lower educational level, were employed, married, and had not smoked cigarettes in the 30 days before the survey (Table 1).

\section{Waterpipe tobacco smoking behaviour and self-reported addiction}

The mean age at starting to smoke waterpipe tobacco in current waterpipe smokers was 18.3 (SD 3.5) years. Most of the respondents $(1210,81.2 \%)$ smoked daily, smoked on average 5.8 (SD 3.5) waterpipe hagar a day, and smoked at least 25 hagar in the 30 days before the survey. Almost half of the participants $(719,48.2 \%)$ reported usually smoking the waterpipe at home/work, although participants reported an average 8.5 (SD 9.1) café visits for waterpipe tobacco smoking in the 30 days before the survey. Most participants usually smoked on their own (1041, 69.9\%), smoked unflavoured tobacco $(1229,82.5 \%)$ and purchased their waterpipe tobacco themselves from markets or smoke shops (797, 53.5\%). Mean daily spending on waterpipe tobacco smoking was 12.0 Egyptian pounds (US\$ 0.7) and $1213(81.4 \%$ ) participants spent $2-10 \%$ of their monthly income on waterpipe tobacco smoking (Table 2).

Significantly more participants who self-reported addiction to waterpipe tobacco smoking than those who did not started waterpipe tobacco smoking at a younger age, were daily smokers, smoked on average more hagar a day and in the 30 days before the survey, usually smoked at home or at work, smoked alone, smoked unflavoured tobacco and purchased their waterpipe tobacco from markets or smoke shops. However, their mean daily spending on waterpipe tobacco smoking and per cent of their monthly income spent on waterpipe tobacco smoking did not differ much from those who did not selfreport addiction to waterpipe tobacco smoking (Table 2).

\section{Perceived harm of waterpipe tobacco smoking and self-reported addiction}

Only 491 (33.0\%) of current waterpipe smokers thought about how much this habit cost them. Two thirds of them $(933,62.6 \%)$ thought waterpipe tobacco smoking was bad for health; however, only 446 (29.9\%) often worried about the health hazards of waterpipe tobacco smoking. Compared with cigarettes, $820(55.0 \%)$ of current waterpipe smokers thought waterpipe tobacco smoking was more harmful and $615(41.3 \%)$ thought waterpipe tobacco con- 


\begin{tabular}{|c|c|c|c|c|}
\hline \multirow[t]{2}{*}{ Characteristic } & \multirow[t]{2}{*}{ Total $(n=1490)$} & \multicolumn{2}{|c|}{$\begin{array}{l}\text { Self-reported addiction to waterpipe } \\
\text { tobacco smoking }\end{array}$} & \multirow[t]{2}{*}{ P-value a } \\
\hline & & No $(n=1106)$ & Yes $(n=384)$ & \\
\hline \multicolumn{5}{|l|}{ Age (years) } \\
\hline Mean (SD) & $35.5(13.5)$ & $33.3(13.7)$ & $41.8(14.1)$ & $<0.001$ \\
\hline \multirow[t]{2}{*}{ Min-max, median (IQR) } & $18-87,35(23-46)$ & $18-75,31.5(22-43)$ & $18-87,42.0(31-54)$ & \\
\hline & No. (\%) & No. (\%) & No. (\%) & P-value ${ }^{b}$ \\
\hline \multicolumn{5}{|l|}{ Age group (years) } \\
\hline $18-24$ & $535(35.9)$ & $447(40.4)$ & $88(22.9)$ & $<0.001$ \\
\hline$\geq 25$ & $955(64.1)$ & $659(59.6)$ & $296(77.1)$ & \\
\hline \multicolumn{5}{|l|}{ Gender } \\
\hline Male & $1361(91.3)$ & $992(89.7)$ & $369(96.1)$ & $<0.001$ \\
\hline Female & $129(8.7)$ & $114(10.3)$ & $15(3.9)$ & \\
\hline \multicolumn{5}{|l|}{ Residence } \\
\hline Rural & $883(59.3)$ & $650(58.8)$ & $233(60.7)$ & 0.547 \\
\hline Urban & $607(40.7)$ & $456(41.2)$ & $151(39.3)$ & \\
\hline \multicolumn{5}{|l|}{ Educational level } \\
\hline $\begin{array}{l}\text { No schooling/primary/ } \\
\text { middle/secondary }\end{array}$ & $604(40.5)$ & $492(44.5)$ & $112(29.2)$ & $<0.001$ \\
\hline Vocational/university & $886(59.5)$ & $614(55.5)$ & $272(70.8)$ & \\
\hline \multicolumn{5}{|l|}{ Occupation } \\
\hline $\begin{array}{l}\text { Unskilled or manual worker/ } \\
\text { student/unemployedc }\end{array}$ & $468(31.4)$ & $385(34.8)$ & $83(21.6)$ & $<0.001$ \\
\hline $\begin{array}{l}\text { Professional/technical/ } \\
\text { skilled }\end{array}$ & $1022(68.6)$ & $721(65.2)$ & $301(78.4)$ & \\
\hline \multicolumn{5}{|l|}{ Marital status } \\
\hline Unmarried & $527(35.4)$ & $458(41.4)$ & $69(18.0)$ & $<0.001$ \\
\hline Married & $963(64.6)$ & $648(58.6)$ & $315(82.0)$ & \\
\hline \multicolumn{5}{|c|}{ Exposure to second-hand smoke at home (cigarettes or waterpipe tobacco smoke) } \\
\hline No & $481(32.3)$ & $351(31.7)$ & $130(33.9)$ & 0.448 \\
\hline Yes & $1009(67.7)$ & $755(68.3)$ & $254(66.1)$ & \\
\hline Crowding index (persons per roo & & & & 0.003 \\
\hline$<2$ & $585(39.3)$ & $407(36.8)$ & $178(46.4)$ & \\
\hline $2-3$ & $885(59.4)$ & $682(61.7)$ & $203(52.9)$ & \\
\hline$>3$ & $20(1.3)$ & $17(1.5)$ & $3(0.8)$ & \\
\hline \multicolumn{5}{|c|}{ Smoked cigarettes in the past 30 days } \\
\hline No & $827(55.5)$ & $581(52.5)$ & $246(64.1)$ & $<0.001$ \\
\hline Yes & $663(44.5)$ & $525(47.5)$ & 138 (35.9) & \\
\hline
\end{tabular}

andependent samples t-test.

${ }^{b}$ Chi-squared test.

'Unemployed includes retired.

P-values $<0.05$ indicate statistically significant differences between current waterpipe smokers who self-reported addiction to waterpipe tobacco smoking and those who did not

tained more nicotine. Significantly fewer participants who self-reported addiction to waterpipe tobacco smoking than those who did not perceive the harms of waterpipe tobacco smoking (Table 3).

\section{Perceived behavioural control of waterpipe tobacco smoking and self-reported addiction}

Of the current waterpipe smokers, 678 (45.5\%) were confident in their ability to quit waterpipe tobacco smoking any time they decided to, although $663(44.5 \%)$ thought quitting waterpipe tobacco smoking permanently would be difficult. Only $290(19.5 \%)$ participants had previously attempted to quit waterpipe tobacco smoking but 953 (64.0\%) intended to quit, although $872(58.5 \%)$ of them had not set a quit date (Table 3).

Significantly fewer participants who self-reported addiction to waterpipe tobacco smoking than those who did not were confident that they could quit waterpipe 
Table 2 Waterpipe tobacco smoking behaviour of current waterpipe smokers who self-reported addiction to waterpipe tobacco smoking compared with those who did not

\begin{tabular}{|c|c|c|c|c|}
\hline \multirow[t]{2}{*}{$\begin{array}{l}\text { Waterpipe Tobacco Smoking } \\
\text { behaviour }\end{array}$} & \multirow[t]{2}{*}{ Total $(n=1490)$} & \multicolumn{2}{|c|}{$\begin{array}{l}\text { Self-reported addiction to waterpipe } \\
\text { tobacco smoking }\end{array}$} & \multirow[t]{2}{*}{ P-value } \\
\hline & & No $(n=1106)$ & Yes $(n=384)$ & \\
\hline \multicolumn{5}{|c|}{ Age at starting waterpipe tobacco smoking (years) } \\
\hline Mean (SD) & $18.3(3.5)$ & $18.6(3.6)$ & $17.6(3.1)$ & $0.002^{\mathrm{a}}$ \\
\hline Min-max, median (IQR) & $11-40,18(16-19)$ & $11-40,18(17-20)$ & $11-35,17(16-18)$ & \\
\hline \multicolumn{4}{|c|}{ Self-reported usual frequency of waterpipe tobacco smoking, No. (\%) } & $<0.001^{c}$ \\
\hline Monthly & $29(1.9)$ & $25(2.3)$ & $4(1.0)$ & \\
\hline Weekly & $251(16.8)$ & $239(21.6)$ & $12(3.1)$ & \\
\hline Daily & $1210(81.2)$ & $842(76.1)$ & $368(95.8)$ & \\
\hline \multicolumn{5}{|l|}{ No. of hagar smoked a day ${ }^{d}$} \\
\hline Mean (SD) & $5.8(3.5)$ & $5.2(3.1)$ & $7.4(4.1)$ & $<0.001^{\mathrm{a}}$ \\
\hline Min-max, median (IQR) & $1-30,6(2-8)$ & $1-20,5(2-8)$ & $1-30,8(4-10)$ & \\
\hline \multicolumn{4}{|c|}{ No. of hagar smoked in the past 30 days, no. $(\%)^{d}$} & $<0.001^{c}$ \\
\hline$<4$ & $24(1.6)$ & $21(1.9)$ & $3(0.8)$ & \\
\hline $4-9$ & $122(8.2)$ & $117(10.6)$ & $5(1.3)$ & \\
\hline $10-14$ & $21(1.4)$ & $19(1.7)$ & $2(0.5)$ & \\
\hline $15-19$ & $38(2.6)$ & $36(3.3)$ & $2(0.5)$ & \\
\hline $20-24$ & $68(4.6)$ & $65(5.9)$ & $3(0.8)$ & \\
\hline$\geq 25$ & $1217(81.7)$ & $848(76.7)$ & $369(96.1)$ & \\
\hline \multicolumn{4}{|c|}{ Usual place to smoke the waterpipe tobacco, no. (\%) } & $<0.001^{c}$ \\
\hline Café/restaurant & $693(46.5)$ & $554(50.1)$ & $139(36.2)$ & \\
\hline At home & $474(31.8)$ & $313(28.3)$ & $161(41.9)$ & \\
\hline Workplace & $245(16.4)$ & $164(14.8)$ & $81(21.1)$ & \\
\hline At a friend's place & $78(5.2)$ & $75(6.8)$ & $3(0.8)$ & \\
\hline \multicolumn{5}{|c|}{ No. of times smoked a waterpipe at a café in the past 30 days } \\
\hline Mean (SD) & $8.5(9.1)$ & $8.6(8.7)$ & $8.1(10.0)$ & $<0.001^{\mathrm{a}}$ \\
\hline Min-max, median (IQR) & $0-30,5(0-13)$ & $0-30,6(0-12)$ & $0-30,3(0-15)$ & \\
\hline \multicolumn{4}{|c|}{ Usually smokes waterpipe alone, No. (\%) ${ }^{b}$} & $<0.001^{\mathrm{c}}$ \\
\hline No & $449(30.1)$ & $378(34.2)$ & $71(18.5)$ & \\
\hline Yes & $1041(69.9)$ & $728(65.8)$ & $313(81.5)$ & \\
\hline \multicolumn{4}{|c|}{ Type of waterpipe tobacco usually smoked, No. (\%) ${ }^{b}$} & $0.002^{c}$ \\
\hline Flavoured & $261(17.5)$ & $213(19.3)$ & $48(12.5)$ & \\
\hline Unflavoured & $1229(82.5)$ & $893(80.7)$ & $336(87.5)$ & \\
\hline \multicolumn{4}{|c|}{ Usual source of waterpipe tobacco, No. (\%) ${ }^{b}$} & $0.001^{c}$ \\
\hline Market/street vendor/smoke sh & $797(53.5)$ & $561(50.7)$ & $236(61.5)$ & \\
\hline Café/restaurant & $662(44.4)$ & $524(47.4)$ & $138(35.9)$ & \\
\hline Friend or relative & $11(0.7)$ & $9(0.8)$ & $2(0.5)$ & \\
\hline Internet/other & $20(1.3)$ & $12(1.1)$ & $8(2.1)$ & \\
\hline \multicolumn{5}{|c|}{ Amount spent a day on waterpipe tobacco smoking (Egyptian pounds) ${ }^{e}$} \\
\hline Mean (SD) & $12.0(17.8)$ & $11.9(17.3)$ & $12.5(19.0)$ & $0.421^{\mathrm{a}}$ \\
\hline Min-max, median (IQR) & $1-100,5(3-10)$ & $1-100,4(3-12)$ & $1-100,4(1-5)$ & \\
\hline \multicolumn{4}{|c|}{ Percentage of monthly income spent on waterpipe tobacco smoking, No. (\%) } & $0.037^{c}$ \\
\hline$\leq 1$ & $194(13.0)$ & $147(13.3)$ & $47(12.2)$ & \\
\hline $2-10$ & $1213(81.4)$ & $893(80.7)$ & $320(83.3)$ & \\
\hline $11-50$ & $81(5.4)$ & $66(6.0)$ & $15(3.9)$ & \\
\hline$>50$ & $2(0.1)$ & $0(0.0)$ & $2(0.5)$ & \\
\hline
\end{tabular}

andependent samples t-test.

${ }^{b}$ Percentages may not sum to $100 \%$ because of rounding.

'Chi-squared test.

${ }^{d}$ Waterpipe tobacco portion.

US\$ $1=17.545$ Egyptian pounds (14).

P-values $<0.05$ indicate statistically significant differences between current waterpipe smokers who self-reported addiction to waterpipe tobacco smoking and those who did not. 


\begin{tabular}{|c|c|c|c|c|}
\hline \multirow[t]{3}{*}{$\begin{array}{l}\text { Perceived harm and perceived behavioural } \\
\text { control of waterpipe tobacco smoking }\end{array}$} & \multirow{3}{*}{ 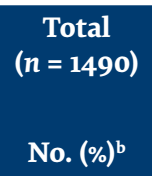 } & \multicolumn{2}{|c|}{$\begin{array}{l}\text { Self-reported addiction to waterpipe } \\
\text { tobacco smoking }\end{array}$} & \multirow[t]{3}{*}{ P-value } \\
\hline & & No $(n=1106)$ & Yes $(n=384)$ & \\
\hline & & No. $(\%)^{b}$ & No. $(\%)^{b}$ & \\
\hline Think about the cost of waterpipe tobacco smoking & & & & $<0.001$ \\
\hline Never & $999(67.0)$ & $686(62.0)$ & $313(81.5)$ & \\
\hline Sometimes & $431(28.9)$ & $369(33.4)$ & $62(16.1)$ & \\
\hline Often & $60(4.0)$ & $51(4.6)$ & $9(2.3)$ & \\
\hline Worry about health hazards of waterpipe tobacco smoking & & & & $<0.001$ \\
\hline Never & $394(26.4)$ & $211(19.1)$ & $183(47.7)$ & \\
\hline Sometimes & $650(43.6)$ & $514(46.5)$ & $136(35.4)$ & \\
\hline Often & $446(29.9)$ & $381(34.4)$ & $65(16.9)$ & \\
\hline In general, effect of waterpipe tobacco smolking on health is: & & & & 0.016 \\
\hline Good & $177(11.9)$ & $125(11.3)$ & $52(13.5)$ & \\
\hline Neither good nor bad & $311(20.9)$ & $239(21.6)$ & $72(18.8)$ & \\
\hline Bad & $933(62.6)$ & $681(61.6)$ & $252(65.6)$ & \\
\hline Don't know & $69(4.6)$ & $61(5.5)$ & $8(2.1)$ & \\
\hline Compared with cigarettes, waterpipe tobacco smoking is: & & & & 0.003 \\
\hline Less harmful & $243(16.3)$ & $195(17.6)$ & $48(12.5)$ & \\
\hline About the same harm & $329(22.1)$ & $221(20.0)$ & $108(28.1)$ & \\
\hline More harmful & $820(55.0)$ & $614(55.5)$ & $206(53.6)$ & \\
\hline Don't know & $98(6.6)$ & $76(6.9)$ & $22(5.7)$ & \\
\hline Compared with cigarettes, waterpipe tobacco contains: & & & & $<0.001$ \\
\hline Less nicotine & $234(15.7)$ & $185(16.7)$ & $49(12.8)$ & \\
\hline About the same amount of nicotine & $245(16.4)$ & $182(16.5)$ & $63(16.4)$ & \\
\hline More nicotine & $615(41.3)$ & $493(44.6)$ & $122(31.8)$ & \\
\hline Don't know & $396(26.6)$ & $246(22.2)$ & $150(39.1)$ & \\
\hline Am confident I can quit waterpipe tobacco smoking & & & & $<0.001$ \\
\hline No & $812(54.5)$ & $477(43.1)$ & $335(87.2)$ & \\
\hline Yes & $678(45.5)$ & $629(56.9)$ & $49(12.8)$ & \\
\hline Quitting waterpipe tobacco smoking is: & & & & $<0.001$ \\
\hline Easy & $409(27.4)$ & $396(35.8)$ & $13(3.4)$ & \\
\hline Difficult & $663(44.5)$ & $350(31.6)$ & $313(81.5)$ & \\
\hline Don't know & $418(28.1)$ & $360(32.5)$ & $58(15.1)$ & \\
\hline Have tried to quit waterpipe tobacco smoking before ${ }^{c}$ & & & & $<0.001$ \\
\hline No & $1194(80.5)$ & $845(76.7)$ & $349(91.4)$ & \\
\hline Yes & $290(19.5)$ & $257(23.3)$ & $33(8.6)$ & \\
\hline Intend to quit waterpipe tobacco smoking & & & & $<0.001$ \\
\hline Not at all & $537(36.0)$ & $261(23.6)$ & 276 (71.9) & \\
\hline In the next month & $12(0.8)$ & $11(1.0)$ & $1(0.3)$ & \\
\hline In the next 6 months & $69(4.6)$ & $57(5.2)$ & $12(3.1)$ & \\
\hline In the future & $872(58.5)$ & $777(70.3)$ & $95(24.7)$ & \\
\hline
\end{tabular}

${ }^{a}$ Chi-squared test.

${ }^{b}$ Percentages may not sum to $100 \%$ because of rounding.

'Contains some missing values, $n=1484$.

P-values $<0.05$ indicate statistically significant differences between current waterpipe smokers who self-reported addiction to waterpipe tobacco smoking and those who did not. 


\begin{tabular}{|c|c|c|c|c|c|c|}
\hline \multirow[t]{2}{*}{ Variable } & \multicolumn{2}{|c|}{ Univariate analysis } & \multicolumn{4}{|c|}{ Multivariable logistic regression analysis } \\
\hline & OR $(95 \% \mathrm{CI})$ & $P$-value & $\boldsymbol{\beta}$ & SE & P-value & ORa $(95 \% \mathrm{CI})$ \\
\hline Age group ( $\geq 25$ years) & $2.3(1.8-3.0)$ & $<0.001$ & 0.000 & 0.218 & 0.999 & $1.0(0.7-1.5)$ \\
\hline Gender (male) & $2.8(1.6-4.9)$ & $<0.001$ & 0.098 & 0.329 & 0.766 & $1.1(0.6-2.1)$ \\
\hline Education (vocational/university) & $2.0(1.5-2.5)$ & $<0.001$ & 0.16 & 0.179 & 0.370 & $1.2(0.8-1.7)$ \\
\hline $\begin{array}{l}\text { Occupation (professional/technical/ } \\
\text { skilled) }\end{array}$ & $1.9(1.5-2.5)$ & $<0.001$ & 0.166 & 0.167 & 0.321 & $1.2(0.9-1.6)$ \\
\hline Marital status (married) & $3.2(2.4-4.3)$ & $<0.001$ & 0.600 & 0.23 & 0.009 & $1.8(1.2-2.9)$ \\
\hline Current cigarette smoking (no) & $1.3(1.3-2.1)$ & $<0.001$ & 0.219 & 0.139 & 0.114 & $1.3(0.9-1.6)$ \\
\hline $\begin{array}{l}\text { Age at starting waterpipe tobacco } \\
\text { smoking (<18 years) }\end{array}$ & $2.5(2.0-3.2)$ & $<0.001$ & 0.796 & 0.141 & $<0.001$ & $2.2(1.7-2.9)$ \\
\hline $\begin{array}{l}\text { Frequency of smoking the waterpipe } \\
\text { tobacco (daily) }\end{array}$ & $7.2(4.3-12.1)$ & $<0.001$ & 0.677 & 0.299 & 0.024 & $2.0(1.1-3.5)$ \\
\hline $\begin{array}{l}\text { Place where waterpipe tobacco } \\
\text { smoked (at home) }\end{array}$ & $1.8(1.4-2.3)$ & $<0.001$ & 0.428 & 0.251 & 0.087 & $1.5(0.9-2.5)$ \\
\hline $\begin{array}{l}\text { Company when smoking the } \\
\text { waterpipe tobacco(none, smokes } \\
\text { alone) }\end{array}$ & $2.3(1.7-3.1)$ & $<0.001$ & 0.689 & 0.17 & $<0.001$ & $2.0(1.4-2.8)$ \\
\hline $\begin{array}{l}\text { Type of waterpipe tobacco smoked } \\
\text { (unflavoured) }\end{array}$ & $1.7(1.2-2.3)$ & $<0.001$ & 0.476 & 0.244 & 0.051 & $1.6(1.0-2.6)$ \\
\hline $\begin{array}{l}\text { Source of waterpipe tobacco (self- } \\
\text { purchase) }\end{array}$ & $1.6(1.3-2.1)$ & $<0.001$ & 0.139 & 0.255 & 0.585 & $1.2(0.7-1.9)$ \\
\hline $\begin{array}{l}\text { Monthly amount spent on waterpipe } \\
\text { tobacco smoking ( } \geq 150 \text { Egyptian } \\
\text { pounds, US\$ } 8.6 \text { ) }\end{array}$ & $3.4(2.6-4.3)$ & $<0.001$ & 1.397 & 0.162 & $<0.001$ & $4.1(2.9-5.6)$ \\
\hline Constant & & & -4.758 & 0.379 & $<0.001$ & 0.009 \\
\hline
\end{tabular}

$\mathrm{OR}=$ odds ratio $\mathrm{CI}=$ confidence interval; $\mathrm{SE}=$ standard error; $\mathrm{OR} a=$ adjusted odds ratio.

tobacco smoking (Table 3). In addition, significantly more participants who self-reported addiction to waterpipe tobacco smoking than those who did not thought quitting waterpipe tobacco smoking would be difficult, and significantly fewer participants who self-reported addiction to waterpipe tobacco smoking than those who did not had ever attempted to quit. Furthermore, significantly more participants who self-reported addiction to waterpipe tobacco smoking had no intention of quitting waterpipe tobacco smoking compared with those who did not self-report addiction (Table 3).

\section{Determinants of self-reported addiction to waterpipe tobacco smoking}

Table 4 shows results of the univariate and multivariable logistic regression analyses. Of the variables tested in the multivariable analysis for their association with self-reported addiction to waterpipe tobacco smoking, the following were statistically significant independent determinants of self-reported addiction: younger age at starting waterpipe tobacco smoking $(\mathrm{ORa}=2.2$, $95 \%$ CI: 1.7-2.9), daily use of waterpipe tobacco (ORa $=2.0$, 95\% CI: 1.1-3.5), usually smoking the waterpipe alone $(\mathrm{ORa}=2.0$, 95\% CI: 1.4-2.8), being married (ORa $=1.8$, 95\% CI: 1.2-2.9), and monthly spending on waterpipe tobacco smoking of $\geq 150$ Egyptian pounds (US\$ 8.6) $(\mathrm{ORa}=4.1,95 \%$ CI: 2.9-5.6) (Table 4).

\section{Discussion}

This study is among the few that have assessed self-reported addiction to waterpipe tobacco smoking. A quarter $(25.8 \%)$ of current waterpipe smokers in the study self-reported addiction to waterpipe tobacco smoking. Compared with waterpipe smokers who did not consider themselves addicted to waterpipe tobacco smoking, those who self-reported addiction started smoking the waterpipe tobacco at a younger age, smoked it more frequently and in greater amounts, and were more likely to smoke the waterpipe tobacco at home or at work and smoke alone. Participants who self-reported addiction to waterpipe tobacco smoking also reported more difficulty to quit, lower self-efficacy, fewer quit attempts, less intention to quit and less perceived harm of waterpipe tobacco smoking. In addition, being married, and monthly spending of $\geq 150$ Egyptian pounds (US\$8.6) on waterpipe tobacco smoking were independent determinants of self-reported addiction to waterpipe tobacco smoking. However, waterpipe tobacco smoking was generally affordable for all current waterpipe smokers.

Only a few studies have explored dependence on waterpipe tobacco smoking using a direct question about self-reported addiction $(15,16)$. In line with the findings of this study, studies from The Syrian Arab Republic and the United States of America (USA) reported that the subjective perception of smokers of being addicted was 
associated with higher frequency of waterpipe tobacco smoking $(15,16)$. Evidence of this association from other low- and middle-income countries that are experiencing an increase in waterpipe tobacco smoking is needed. There are features unique to waterpipe tobacco smoking that influence the development and manifestations of tobacco dependence (1). Waterpipe smokers may not be aware of these features (1). For example, a considerable proportion $(28.1 \%)$ of waterpipe smokers in the present study simply did not know whether they were addicted to waterpipe tobacco. This is an important finding that reflects a lack of knowledge about the harms of waterpipe tobacco smoking which leads to uncertainty about the dependence waterpipe tobacco smoking can cause. Future or potential perceived addiction to waterpipe tobacco has been investigated in recent studies in young people in the USA and Lebanon (17-19). Participants reported low perceived addictiveness of waterpipe tobacco smoking and low perceived chances of becoming addicted (17-19).

Younger age at starting waterpipe tobacco smoking, daily waterpipe tobacco smoking, and usually smoking the waterpipe tobacco alone were independent determinants of self-reported addiction to waterpipe tobacco smoking in the current study. These findings are in line with earlier studies $(15,16,20,21)$. Another important finding of the present study is that waterpipe tobacco smoking is not intermittent or occasional, which was also found in recent Egyptian national and population-based surveys $(6,9)$. Furthermore, the transition from social waterpipe tobacco use to an individual pattern of use has been considered an indicator of waterpipe tobacco dependence (1). Although none of the sociodemographic characteristics examined in this study independently determined self-reported addiction to waterpipe tobacco smoking, except for being married, previous qualitative studies in Egypt suggest waterpipe tobacco smoking is more widely and intensively done in rural settings $(22,23)$. Further investigations are needed to confirm whether there are other sociodemographic differences associated with self-reported addiction to waterpipe tobacco smoking.

Waterpipe tobacco smoking was generally affordable for all participants who were current waterpipe smokers. A mean monthly spending of $\geq 150$ Egyptian pounds (US\$ 8.6) on waterpipe tobacco smoking was the strongest independent determinant of self-reported addiction to waterpipe tobacco smoking. This finding has policy implications if we consider that a previous study in 2003-2004 found that current waterpipe smokers spent on average 8 Egyptian pounds (US\$1.30) a month on waterpipe tobacco smoking (24). The six-fold increase in spending over these 15 years may be due to increase in consumption (even when considering inflation and other factors). On the other hand, the taxation policy for waterpipe tobacco has not been scaled up to face this change in waterpipe tobacco smoking behaviour. Egypt implements the highest taxation measures recommended by the WHO for cigarettes. However, unfortunately, this successful policy is not implemented on waterpipe tobacco. According to the WHO report on the global tobacco epidemic 2019, the total tax on cigarettes (pack of 20 ) is $77.1 \%$, while the total tax on a pack of waterpipe tobacco (20 g) is only $39.4 \%(25)$.

Participants in the present study who self-reported addiction to waterpipe tobacco smoking thought it more difficult to quit, had lower self-efficacy (were not confident in their ability to quit), made fewer attempts to quit, had less intention to quit, and perceived less harm from waterpipe tobacco smoking than those who did not think they were addicted. Similar evidence has been reported in previous studies; most motivated waterpipe smokers who wanted to quit were unable to do so or had attempted to quit but had been unsuccessful (1). These waterpipe smokers may show an indicator of tobacco dependence, which is failed efforts to reduce or control substance use. There was also an inverse relationship between perceived self-efficacy and perceived addiction to waterpipe tobacco smoking, a finding also reported in an earlier study (15).

Tobacco control efforts have long neglected waterpipe tobacco smoking. Therefore, tobacco users instinctively associate smoking or the harms of smoking with cigarette smoking but not waterpipe tobacco smoking (1). This is because waterpipe tobacco smoking is not highlighted as much as cigarettes in national tobacco control policies, whether in smoke-free policies, tobacco taxation or education interventions. This may have indirectly contributed to the misbeliefs that waterpipe tobacco smoking is less harmful, addictive and deadly than cigarette smoking.

This study had limitations. First, the cross-sectional study design and the non-random sampling limits the generalizability of results and it is not possible to attribute causality for the observed associations. Second, the measures assessed relied on self-reporting, which may be subject to social desirability and recall bias due to the administration of the interview survey. However, selfreports of smoking status have been argued to be valid (26). More importantly, self-reporting of dependence is an essential milestone in the behavioural change stages and in assessing a smoker's readiness to change. Third, no formal dependence measures or laboratory tests were used in this study which could have more accurately determine nicotine dependence. This was not feasible in the study design, hence the patterns of waterpipe tobacco smoking behaviour that are known to be associated with dependence were examined. In addition, standardized measures of waterpipe-specific dependence are still being developed, refined and tested for potential use in different cultures $(1,20,27)$. Finally, this study did not address the length of the waterpipe tobacco smoking session. The length of session has been reported to be associated with progressive nicotine dependence in waterpipe smokers (28). Nonetheless, the relatively large sample in the different subgroups provided enough observations for comparisons to be made and may have minimized potential biases in the results. 


\section{Conclusion}

Surveillance efforts should estimate the national prevalence of dependence on waterpipe tobacco to provide clarity on waterpipe tobacco-induced dependence. Comprehensive waterpipe-specific interventions, including education, taxation and cessation, are urgently needed. The fact that a considerable proportion of waterpipe smokers were uncertain whether they were addicted to waterpipe tobacco smoking highlights the need to more effectively disseminate evidence-based waterpipe-specific health education messages and incorporate this infor- mation in ongoing public health campaigns. The affordability of waterpipe tobacco smoking indicates the need to reform waterpipe-specific tobacco taxation, particularly as despite a six-fold higher monthly expenditure on waterpipe tobacco smoking compared with earlier reports, waterpipe smokers who self-identified as addicted to waterpipe tobacco smoking continued to smoke waterpipe tobacco. Strengthening cessation-seeking behaviour and interventions focusing on the complexity of perceived self-efficacy and perceived addiction to waterpipe tobacco smoking in all levels of health care services is another important policy approach to control tobacco use.

\section{Acknowledgements}

Thanks to the participants of this study, and the field and data management teams for their commitment to quality in conducting the research.

Funding: This work was funded by the International Development Research Centre, Ottawa, Canada (Grant 106981-001) through the American University of Beirut, the Tobacco Control Research Group. The funders had no role in study design, data collection and analysis, decision to publish or preparation of the manuscript.

Competing interests: None declared.

\section{Dépendance auto-déclarée et contrôle perçu du tabagisme par pipe à eau et comportements associés en Égypte : implications au niveau des politiques}

\section{Résumé}

Contexte : Les études sur la dépendance au tabagisme par pipe à eau sont limitées.

Objectifs : La présente étude visait à évaluer la dépendance auto-déclarée au tabagisme par pipe à eau chez les fumeurs égyptiens et à identifier les facteurs sociodémographiques, le contrôle perçu et les comportements tabagiques associés.

Méthodes: Des enquêtes transversales ont été menées auprès d'adultes égyptiens en 2015 et 2017. les données sur 1490 fumeurs de pipe à eau, au moment de l'étude, ont été analysées, y compris les caractéristiques démographiques, le comportement tabagique du tabagisme par pipe à eau (âge au début de l'habitude, fréquence, quantité, compagnie, lieu du tabagisme et dépenses), les préjudices perçus de cette consommation ainsi que la dépendance auto-déclarée au tabagisme par pipe à eau et le contrôle perçu de ce type de tabagisme (capacité d'arrêter, difficulté à arrêter, tentatives d'arrêter et intention d'arrêter).

Résultats : Un quart $(25,8 \%)$ des participants ont déclaré une dépendance au tabagisme par pipe à eau (hommes $27,1 \%$, femmes 11,6\%). Ces participants ont déclaré avoir moins de confiance en leur capacité à arrêter de fumer, moins de tentatives de sevrage, moins d'intention d'arrêter et moins de préjudices perçus de cette consommation que ceux qui n'en étaient pas dépendants $(p<0,001)$. Les variables associées à la dépendance auto-déclarée étaient les suivantes : âge plus jeune au début de l'habitude du tabagisme par pipe à eau (odds ratio ajusté (ORa) $=2,2$, intervalle de confiance à $95 \%$ (IC à $95 \%$ ) : 1,7-2,9), tabagisme quotidien par pipe à eau (ORa $=2,0$, IC à $95 \%: 1,1-3,5)$, le fait de fumer seul (ORa $=2,0$, IC à $95 \%: 1,4-2,8$ ), le fait d'être marié (ORa $=1,8$, IC à $95 \%: 1,2-2,9)$ et des dépenses mensuelles pour fumer des pipes à eau supérieures ou égales à 150 livres égyptiennes (8,6 USD) (ORa $=4,1$, IC à $95 \%: 2,9-5,6$ ).

Conclusions : Il est nécessaire de mettre en place des interventions politiques de grande envergure, notamment des programmes d'éducation sur la dépendance au tabagisme par pipe à eau, une taxation accrue pour réduire l'accessibilité financière du tabac pour pipe à eau et des programmes de sevrage tabagique traitant de l'auto-efficacité perçue et la dépendance au tabagisme par pipe à eau.

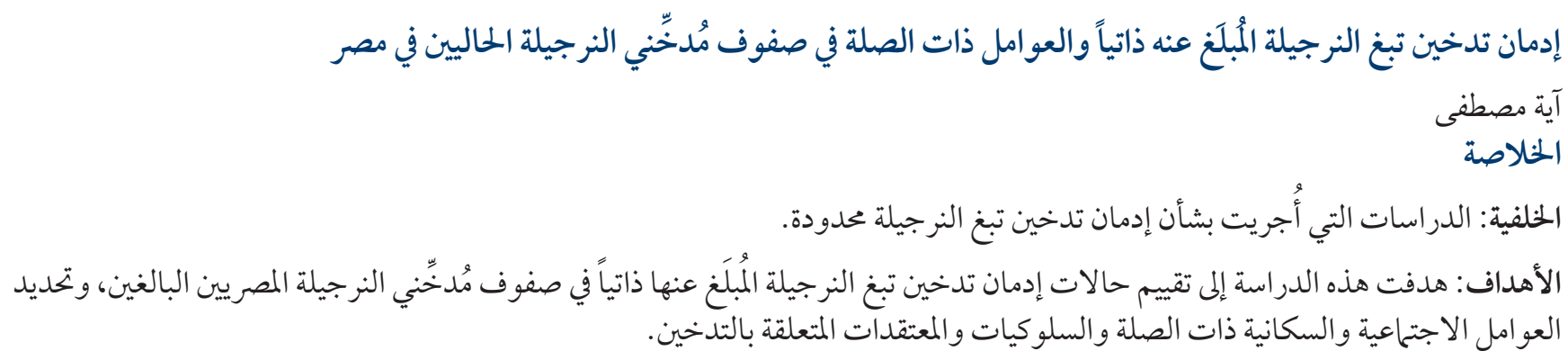




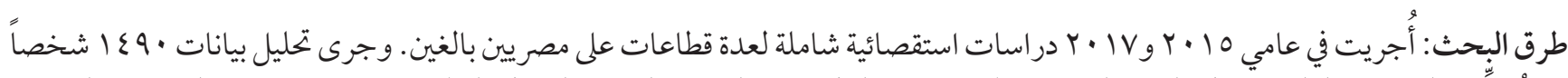

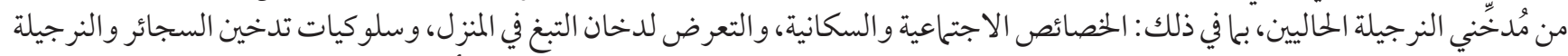

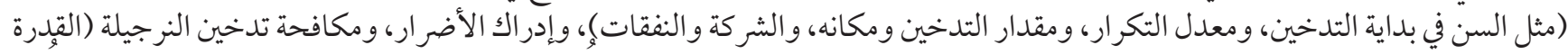

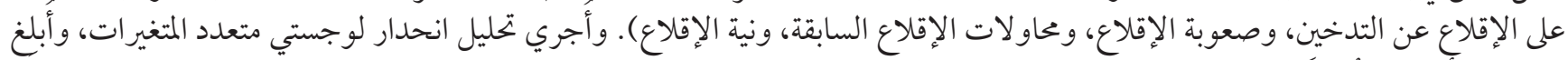

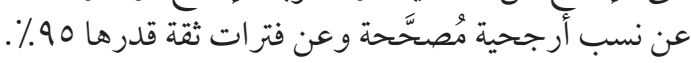

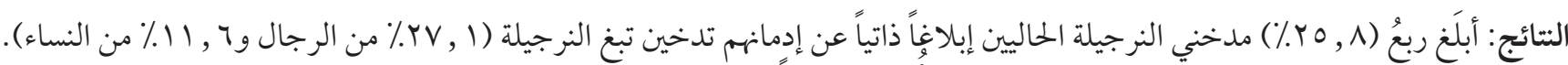

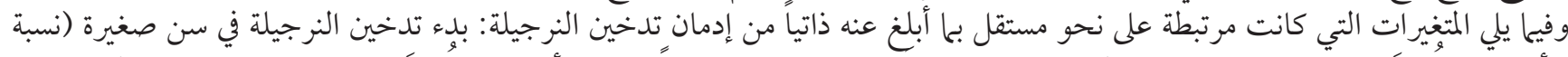

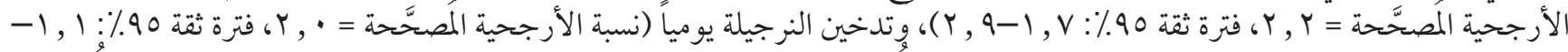

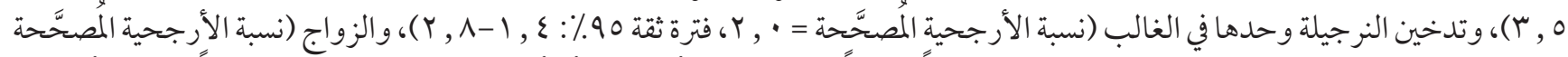

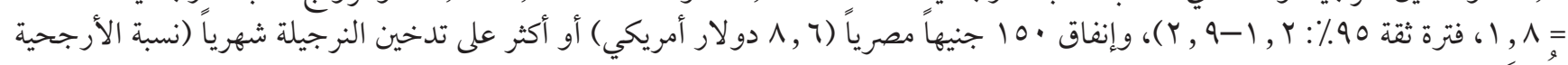

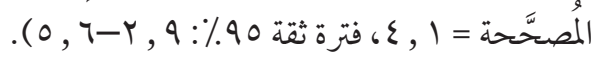

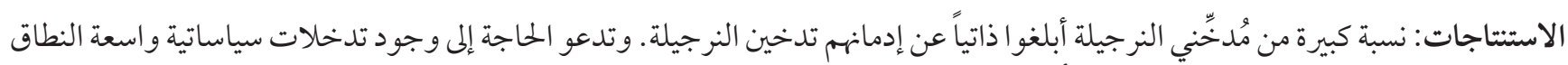

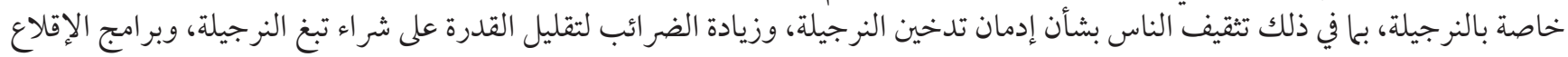
عن التدخين.

\section{References}

1. Aboaziza E, Eissenberg T. Waterpipe tobacco smoking: what is the evidence that it supports nicotine/tobacco dependence? Tob Control. 2015;24 Suppl 1:i44-i53. https://doi.org/10.1136/tobaccocontrol-2014-051910

2. Waziry R, Jawad M, Ballout RA, Al Akel M, Akl EA The effects of waterpipe tobacco smoking on health outcomes: An updated systematic review and meta-analysis. Int J Epidemiol. 2017;46(1):32-43. https://doi.org/10.1093/ije/dywo21

3. Jawad M, Charide R, Waziry R, Darzi A, Ballout RA, Akl EA. The prevalence and trends of waterpipe tobacco smoking: A systematic review. PLoS One. 2018;13(2):e0192191. https://doi.org/10.1371/journal.pone.0192191

4. News. Noncommunicable diseases. Countries commit to achieving 30\% reduction in tobacco use by 2025 . World Health Organization Regional Office for the Eastern Mediterranean (http://www.emro.who.int/noncommunicable-diseases/highlights/countries-commit-to-achieving-30-reduction-in-tobacco-use-by-2025.html, accessed 25 February 2019).

5. WHO global report on trends in prevalence of tobacco smoking 2015. Geneva: World Health Organization; 2015.

6. Egypt National STEPwise Survey for Noncommunicable Diseases Risk Factors Report 2017. A joint report by the Egyptian Ministry of Health and Population and the World Health Organization; 2017 (https://www.who.int/ncds/surveillance/steps/Egypt_National_STEPwise_Survey_For_Noncommunicable_Diseases_Risk_Factors_2017_Report.pdf?ua=1, accessed 25 February 2019)

7. El Awa F, Fouad H, El Naga RA, Emam AH, Labib S. Prevalence of tobacco use among adult and adolescent females in Egypt. East Mediterr Health J. 2013;19(8):749-54.

8. Mostafa A, El Houssine M, Fotouh A. Multiple tobacco use among young adult waterpipe smokers in Egypt. East Mediterr Health J. 2018;24(1):7-17. https://doi.org/10.26719/2018.24.1.17

9. Mostafa A, Mohammed HT, Hussein RS, Hussein WM, Elhabiby M, Safwat W, et al. Do pictorial health warnings on waterpipe tobacco packs matter? Recall effectiveness among Egyptian waterpipe smokers \& non-smokers. PLoS One. 2018;13(12): e0208590. https://doi.org/10.1371/journal.pone.0208590

10. Maynard OM, Gage SH, Munafò MR. Are waterpipe users tobacco-dependent? Addiction. 2013;108(11):1886-7. https://doi. org/10.1111/add.12317

11. Prochaska JO, DiClemente CC, Norcross JC. In search of how people change: applications to addictive behaviors. Am Psychol.1992;47(9):1102-14. https://doi.org/10.1037//0003-066x.47.9.1102

12. Control and prevention of waterpipe tobacco products. Report by the Convention secretariat. World Health Organization Framework Convention on Tobacco Control; 18 July 2014 (http://apps.who.int/gb/fctc/PDF/cop6/FCTC_COP6_11-en.pdf/, accessed 25 February 2019).

13. Maziak W, Ben Taleb Z, Jawad M, Afifi R, Nakkash R, Akl EA, et al. Expert Panel on Waterpipe Assessment in Epidemiological Studies. Consensus statement on assessment of waterpipe smoking in epidemiological studies. Tob Control. 2017;26(3):338-343. https://doi.org/10.1136/tobaccocontrol-2016-052958

14. Xe. Current and historical rate tables. Egyptian pound, 25 February 2019 (https://www.xe.com/currencytables/?from=EGP\&date=2019-02-25, accessed 12 October 2019).

15. Maziak W, Ward KD, Eissenberg T. Factors related to frequency of narghile (waterpipe) use: the first insights on tobacco dependence in narghile users. Drug Alcohol Depend. 2004;76(1):101-6. https://doi.org/10.1016/j.drugalcdep.2004.04.007

16. Ward KD, Eissenberg TE, Gray JN, Srinivas V, Wilson N, Maziak W. Characteristics of US waterpipe users: a preliminary report. Nicotine Tob Res 2007;9(12):1339-46. https://doi.org/10.1080/14622200701705019 
17. Mays D, Tercyak KP, Rehberg K, Crane M, Lipkus IM. Young adult waterpipe tobacco users' perceived addictiveness of waterpipe tobacco. Tob Prev Cessation. 2017;3:133. https://doi.org/10.18332/tpc/80133

18. Lipkus IM, Mays D. Comparing harm beliefs and risk perceptions among young adult waterpipe tobacco smokers and nonsmokers: Implications for cessation and prevention. Addict Behav Rep. 2018;7:103-110. https://doi.org/10.1016/j.abrep.2018.03.003

19. Bahelah R, DiFranza JR, Ward KD, Fouad FM, Eissenberg T, Ben Taleb Z, et al. Correlates of nicotine dependence among adolescent waterpipe smokers. Drug Alcohol Depend. 2016;168:230-8. https://doi.org/10.1016/j.drugalcdep.2016.09.019

20. Primack BA, Khabour OF, Alzoubi KH, Switzer GE, Shensa A, Carroll MV, et al. The LWDS-10J: reliability and validity of the Lebanon Waterpipe Dependence Scale among university students in Jordan. Nicotine Tob Res. 2014;16(7):915-22. https://doi. org/10.1093/ntr/ntuoo2

21. Kassim S, Al-Bakri A, Al'Absi M, Croucher R. Waterpipe tobacco dependence in UK male adult residents: a cross-sectional study. Nicotine Tob Res. 2014;16(3):316-25. https://doi.org/10.1093/ntr/ntt148

22. Mostafa A, Mohammed HT, Hussein WM, Elhabiby M, Safwat W, Labib S, et al. Would placing pictorial health warnings on waterpipe devices reduce waterpipe tobacco smoking? A qualitative exploration of Egyptian waterpipe smokers' and non-smokers' responses. Tob Control. 2019;28(4):475-8. https://doi.org/10.1136/tobaccocontrol-2018-054494

23. Mostafa A, Mohammed HT, Hussein WM, Elhabiby M, Safwat W, Labib S, et al. Plain packaging of waterpipe tobacco? A qualitative analysis exploring waterpipe smokers' and non-smokers' responses to enhanced versus existing pictorial health warnings in Egypt. BMJ Open 2018;8(10):e023496. https://doi.org/10.1136/bmjopen-2018-023496

24. Auf RA, Radwan GN, Loffredo CA, El Setouhy M, Israel E, Mohamed MK. Assessment of tobacco dependence in waterpipe smokers in Egypt. Int J Tuberc Lung Dis. 2012;16(1):132-7. https://doi.org/10.5588/ijtld.11.0457

25. WHO report on the global tobacco epidemic 2019. World Health Organization; 2019 (https://www.who.int/tobacco/global_report/ en/, accessed 26 November 2019).

26. O'Loughlin J, DiFranza JR, Tarasuk J, Meshefedjian G, McMillan-Davey E, Paradis G, et al. Assessment of nicotine dependence symptoms in adolescents: a comparison of five indicators. Tob Control. 2002;11(4):354-60. http://dx.doi.org/10.1136/tc.11.4.354

27. Salameh P, Waked M, Aoun Z. Waterpipe smoking: construction and validation of the Lebanon Waterpipe Dependence Scale (LWDS-11). Nicotine Tob Res. 2008;10(1):149-58. https://doi.org/10.1080/14622200701767753

28. Bahelah R, Ward KD, Ben Taleb Z, DiFranza JR, Eissenberg T, Jaber R, et al. Determinants of progression of nicotine dependence symptoms in adolescent waterpipe smokers. Tob Control. 2018;28(3):254-60. https://doi.org/10.1136/tobaccocontrol-2018-054244 